\title{
The Impact of Nanochloroquine on Restoration of Hepatic and Splenic Mitochondrial Damage against Rodent Malaria
}

\author{
Satyajit Tripathy, Sabyasachi Das, Sandeep Kumar Dash, \\ Sourav Chattopadhyay, and Somenath Roy
}

Immunology and Microbiology Laboratory, Department of Human Physiology with Community Health, Vidyasagar University, Midnapore West Bengal 721102, India

Correspondence should be addressed to Somenath Roy; sroy.vu@hotmail.com

Received 29 January 2013; Accepted 23 April 2013

Academic Editor: Vijaya Rangari

Copyright (C) 2013 Satyajit Tripathy et al. This is an open access article distributed under the Creative Commons Attribution License, which permits unrestricted use, distribution, and reproduction in any medium, provided the original work is properly cited.

The applications of nanotechnology to pharmacology are the potential appliance of biodegradable polymers and convectionenhanced drug delivery in the diagnostics and treatment of diseases. Chitosan is a natural polysaccharide that has attracted significant scientific interest during the last two decades. The present study was to evaluate the possible effects of chitosan tripolyphosphate conjugated nanochloroquine against Plasmodium berghei infection on select makers of oxidative damage and antioxidant status in mitochondria of liver and spleen. P. berghei infection was developed in Swiss mice by intraperitoneal injection of $200 \mu \mathrm{L}$ of infected blood. Parasite-infected mice were treated with chloroquine and nanoconjugated chloroquine. Superoxide radical generation, nitrate level, and oxidized glutathione were increased significantly $(P<0.05)$ in the mitochondria of infected group as compared to control group, and reduced glutathione level, activity of SOD, GPx, GR, and GST, and mitochondrial transmembrane potential were decreased significantly $(P<0.05)$, which were increased or decreased significantly $(P<0.05)$ near to normal in nanoconjugated chloroquine treated group than chloroquine treated group. So, the findings may suggest the advantageous role of nanoconjugated chloroquine against the $P$. berghei induced oxidative damage in hepatic and splenic mitochondria.

\section{Introduction}

Despite years of effort, the developments of effective drug against malaria are available. To date, antimalarial drugs remain the major way to treat the disease. Presently, the most effective way of dealing with malaria is the administration of chemotherapeutic agents. Although drug treatments of malaria are currently the best means of disease management, there is an urgent need for the development of structurally novel and effective antimalarial drugs because of increasing resistance to most presently available antimalarial drugs [13].

The multiplication of the malaria parasite in the blood causes the pathology such as anemia, hemolysis, and damage of the essential organs of the host by the parasite products. Like other microbes, $P$. berghei is also a causative agent that should change the normal condition of host by utilizing or protecting defence factors. It is generally accepted that ROS, NO, and peroxynitrite kill intraerythrocytic malarial parasites $[4,5]$, but the parasite protect itself by antioxidant enzyme [6]. Reactive oxygen species are considered mediators of the inflammatory response and tissue damage in malaria. After a short period of development and multiplication, these parasites leave the liver and invade erythrocytes where they multiply by schizogony, undergoing development through ring, trophozoite, and schizont stages that are responsible for malaria pathogenesis [7]. Plasmodium parasites ingest large quantities of hemoglobin and break it down in their food vacuole. The heam prosthetic group of hemoglobin is considered toxic, possibly due to the generation of oxygen radicals. Malaria infection damages several major organs, such as liver, kidney, brain, spleen, heart, and lungs, and severe malaria is characterized by multiorgan failure. Malarial infection induces mitochondrial pathology by inhibiting mitochondrial respiration, dehydrogenases, and transmembrane potential and damaging the ultrastructure as evident from transmission electron microscopic studies. Mitochondrial GSH depletion and formation of protein 
carbonyl indicate that mitochondrial pathology is associated with mitochondrial oxidative stress [8].

Although it is true that the pharmacokinetics of drugs differ between humans and mice, it is also true that all mammalian Plasmodium species have comparable life cycles and are sensitive to the same drugs [9]. The increasing resistance of malaria parasites to available drugs increases the burden of disease and the need to develop new and effective antimalarial agents [10]. The outbreed albino mouse inoculated with $P$. berghei is generally considered to be a valid model for the primary and large scale screening of drugs for eventual use against human malaria [11]. The use of strains of rodent malaria parasites can yield additional information concerning both its potential value against strains of human malaria and the mode of action of a compound.

Nanocarriers may also allow the use of potentially toxic antimalarials which are still under development. Therefore, chitosan, a natural linear biopolyaminosaccharide obtained by alkaline deacetylation of chitin, was used as a nanodrug carrier for the development of a potent antimalarial compound with conjugating the different age-old drug such as pyrimethamine [12], chloroquine [13], and chitosan have no toxic effect on liver, the most detoxifying organ, and has been recognized as biocompatible. It is also reported that chitosan nanoparticles reduced mitochondrial dehydrogenase activity and also inflicted extensive damage to the cell morphology.

Here, we aimed to isolate the mitochondria from liver and spleen and to study its oxidative stress, antioxidant enzymes level, and mitochondrial membrane potential of these vital organs and also compare the efficacy between only drug and nanoconjugated drug against $P$. berghei induced oxidative damage. This study seeks to demonstrate the potency of chitosan tripolyphosphate conjugated nanochloroquine on the mitochondrial oxidative status of Plasmodium berghei infected Swiss mice.

\section{Materials and Methods}

2.1. Chemicals and Reagents. Sodium chloride $(\mathrm{NaCl})$, giemsa, Tris buffer, Triton-X 100, potassium dihydrogen phosphate $\left(\mathrm{KH}_{2} \mathrm{PO}_{4}\right)$, dipotassium hydrogen phosphate $\left(\mathrm{K}_{2} \mathrm{HPO}_{4}\right)$, ethylene diamine tetra acetate (EDTA), sodium hydroxide $(\mathrm{NaOH})$, chloroform, sodium acetate, ammonium acetate, potassium hydroxide $(\mathrm{KOH})$, methanol, Tris- $\mathrm{HCl}$, formaldehyde, alcohol, and diphenylamine (DPA) were procured from Merck Ltd., SRL Pvt. Ltd., Mumbai, India. 5', $5^{\prime}$ dithio (bis)-2-nitrobenzoic acid (DTNB), standard reduced glutathione $(\mathrm{GSH})$, glutathione reductase (GR), NADPH, $\mathrm{Na} 4, \mathrm{NADPH}$, and oxidized glutathione (GSSG) were procured from Sigma (St. Louis, MO, USA). Commercially available histopaque 1077, HEPES, phorbol mirested aceted (PMA), horse heart cytochrome-c were purchased from Sigma Chemical Co., USA. All other chemicals were from Merck Ltd., SRL Pvt., Ltd., Mumbai, India, and were of the highest purity grade available.

2.2. Parasite. The lethal rodent parasite, Plasmodium berghei NK65 strain, was maintained into sex- and age-matched wild type mice by weekly passage by intraperitoneal injection, and blood stage parasites were stored at $-80^{\circ} \mathrm{C}$.

2.3. Animals. Swiss albino mice, aged 6-8 weeks and weighing 20-25 g were kept up in accordance with the guidelines of the National Institute of Nutrition, Indian Council of Medical Research, Hyderabad, India, and approved by the ethical committee of Vidyasagar University. The animals were housed for a week for acclimatization in groups of five in standard polypropylene cages (Tarson) in the departmental animal house with stainless steel top grill. A clean paddy husk was used as a bedding material. The animals were fed on commercial pellet diet and water ad libitum in polypropylene bottles with stainless steel sipper tubes. The animals were maintained under standard conditions of humidity (55-65\%), temperature $\left(22 \pm 3^{\circ} \mathrm{C}\right)$, and light ( $12 \mathrm{~h}$ light/ $12 \mathrm{~h}$ dark cycles). The animals used in this study did not show any sign of malignancy or other pathological processes.

2.4. Preparation of Chitosan-Tripolyphosphate Nanoparticle and Loading of Chloroquine. Chitosan-tripolyphosphate (CS-TPP) nanoparticle preparation by ionotropic gelation and drug loading was followed according to our previous laboratory report [14].

2.5. Particle Characterization. The drug loaded particle characterization was carried out in different techniques like FTIR, DLS, and zeta potential study, and also the in vitro drug toxicity, the actual drug content, and encapsulation efficiency was reported in our previous study [14].

2.6. Parasite Density Determination and Inoculums Preparation. Standard inoculum was prepared from a donor mouse with $P$. berghei parasitized erythrocytes. Infected blood from the donor mouse was obtained by cardiac puncture after anesthesia with chloroform. Parasitaemia was established by microscopic examination of a thin blood film under oil immersion at $\times 100$ magnification and measured as a percentage of infected erythrocytes in fields of 1000 erythrocytes. Each mouse was infected with a standard inoculum of the $10^{6}$ parasitized erythrocyte suspension in phosphate buffered saline $(0.2 \mathrm{~mL})$ from a donor mouse that was prepared based on percentage parasitaemia.

2.7. Experimental Design. Mice were randomized into control, experimental, and treated groups of twenty four (24) animals and in each group six animals (6) were taken. Randomly selected eighteen (18) mice among twenty four mice were challenged by $P$. berghei $(200 \mu \mathrm{L}$ of infected blood containing $1 \times 10^{5}$ parasites) intraperitoneally (i.p.) for 10 days for successive infection development [15]. Other six mice (Group A) were injected with $200 \mu \mathrm{L} 0.9 \mathrm{gm} \% \mathrm{NaCl}$ intraperitoneally (i.p.) and kept isolated from the infected group. After infection development for 10 days, the mice were isolated in three groups and marked as in the following.

Group “A”: Control: Only 0.9 gm\% Nacl;

Group “B”: Infected control: P. berghei infection + $0.9 \mathrm{gm} \% \mathrm{NaCl}$; 
Group "C": CQ treated: P. berghei infection + CQ drug with the concentration of actual drug content (27.36\%) of $250 \mathrm{mg} / \mathrm{Kg}$ bw nanochloroquine [14];

Group "D": Nch treated: P. berghei infection + $250 \mathrm{mg} / \mathrm{kg}$ bw nanoconjugated chloroquine (effective dose) [14].

All the groups were treated for 15 days of the effective duration of Nch [14]. After the experiment was terminated at the end of schedule, all animals were sacrificed, and liver and spleen were collected to isolate mitochondria.

2.8. Isolation of Mitochondria from Liver and Spleen. After decapitation, tissues were excised from experimental mice of different experimental groups and washed with cold normal saline. Washed tissues of mice from each group were perfused with normal saline and were transferred for homogenize. Mitochondria of liver were isolated using the method described by Aprille and Austin [16]. Tissue was homogenized in the ice-cold buffer containing $0.25 \mathrm{M}$ sucrose, $1 \mathrm{mM}$ EDTA, $1 \mathrm{mM}$ Tris-HCl, and $\mathrm{pH}$ 7.4. The homogenate was centrifuged at $600 \times \mathrm{g}$ for $10 \mathrm{~min}$ at $4^{\circ} \mathrm{C}$, and supernatant fraction were collected and further centrifuged $8000 \times \mathrm{g}$ for $20 \mathrm{~min}$ at $4^{\circ} \mathrm{C}$ to pellet mitochondria and after that it was washed with sucrose buffer solution and then used for following parameters determination.

\subsection{Biochemical Estimation}

2.9.1. Superoxide Radical Generation. The superoxide production was measured according to the method of Boveris [17] by the SOD-inhibitable reduction of acetylated cytochrome c. Cytochrome c reduction by generated superoxide was monitored spectrophotometrically at $550 \mathrm{~nm}$ wavelength with or without the addition of $31 \mathrm{M}$ SOD. The superoxide generation assay was performed in room air conditions.

2.9.2. Nitrite (NO) Level. $100 \mu \mathrm{L}$ of Griess reagent (containing 1 part of $1 \%$ sulfanilamide in $5 \%$ phosphoric acid, and 1 part of $0.1 \%$ of $\mathrm{N}-\mathrm{C}-1$ napthyl ethylene diamine dihydrochloride) was added to $100 \mu \mathrm{L}$ of mitochondria, incubated at room temperature for 10 minutes; readings were taken in a UV spectrophotometer at $550 \mathrm{~nm}$ and compared to a sodium nitrite standard curve (values ranging between 0.5 and $25 \mu \mathrm{M}$ ). The levels of NO were expressed as $\mu \mathrm{M} / \mathrm{mg}$ protein [18].

2.9.3. Determination of Superoxide Dismutase (SOD) Activity. SOD activity of mitochondria of liver and spleen was determined from its ability to inhibit the auto-oxidation of pyrogallol according to KarMahapatra et al. [18]. The reaction mixture considered consisted of $50 \mathrm{mM}$ Tris (hydroxymethyl) aminomethane $(\mathrm{pH}$ 8.2), $1 \mathrm{mM}$ diethylen-etriamine penta acetic acid, and $20-50 \mu \mathrm{L}$ of isolated mitochondria of liver and spleen. The reaction was initiated by the addition of $0.2 \mathrm{mM}$ pyrogallol and the absorbance measured kinetically at $420 \mathrm{~nm}$ at $25^{\circ} \mathrm{C}$ for $3 \mathrm{~min}$. SOD activity was expressed as unit/mg protein.
2.9.4. Determination of Reduced Glutathione (GSH) Level. Reduced glutathione estimation was performed by the method of KarMahapatra et al. [18]. The required amount of mitochondria of liver and spleen was mixed with $25 \%$ of TCA and centrifuged at 2,000 $\times \mathrm{g}$ for $15 \mathrm{~min}$ to settle the precipitated proteins. The supernatant was aspirated and diluted to $1 \mathrm{~mL}$ with $0.2 \mathrm{M}$ sodium phosphate buffer ( $\mathrm{pH}$ 8.0). Later, $2 \mathrm{~mL}$ of $0.6 \mathrm{mM}$ DTNB was added. After 10 minutes, the optical density of the yellow-colored complex formed by the reaction of GSH and DTNB (Ellman's reagent) was measured at $405 \mathrm{~nm}$. A standard curve was obtained with standard reduced glutathione. The levels of GSH were expressed as $\mu \mathrm{g}$ of $\mathrm{GSH} / \mathrm{mg}$ protein.

2.9.5. Determination of Oxidized Glutathione (GSSG) Level. The oxidized glutathione level was measured after derivatization of GSH with 2-vinylpyidine according to the method of KarMahapatra et al. [18]. In brief, with $0.5 \mathrm{~mL}$ mitochondria, $2 \mu \mathrm{L}$ of 2 -vinylpyidine was added and incubated for $1 \mathrm{hr}$ at $37^{\circ} \mathrm{C}$. Then, the mixture was deproteinized with $4 \%$ sulfosalicylic acid and centrifuged at $1,000 \times \mathrm{g}$ for $10 \mathrm{~min}$ to settle the precipitated proteins. The supernatant was aspirated and GSSG level was estimated with the reaction of DTNB at $412 \mathrm{~nm}$ in spectrophotometer and calculated with standard GSSG curve. The levels of GSSG were expressed as $\mu \mathrm{g}$ of GSSG/mg protein.

2.9.6. Determination of Glutathione Peroxidase (GPX) Activity. The GPx activity of was evaluated by the method of KarMahapatra et al. [18]. The reaction mixture contained $50 \mathrm{mM}$ potassium phosphate buffer ( $\mathrm{pH}$ 7.0), $1 \mathrm{mM}$ EDTA, $1 \mathrm{mM}$ sodium azide, $0.2 \mathrm{mM} \mathrm{NADPH}, 1 \mathrm{U}$ glutathione reductase, and $1 \mathrm{mM}$ reduced glutathione. The hepatic and splenic mitochondria, after its addition, were allowed to equilibrate for $5 \mathrm{~min}$ at $25^{\circ} \mathrm{C}$. The reaction was initiated by adding $0.1 \mathrm{~mL}$ of $2.5 \mathrm{mM} \mathrm{H}_{2} \mathrm{O}_{2}$. Absorbance at $340 \mathrm{~nm}$ was recorded for $5 \mathrm{~min}$. Values were expressed as nmol of NADPH oxidized to NADP by using the extinction coefficient of $6.2 \times 10^{3} \mathrm{M}^{-1} \mathrm{~cm}^{-1}$ at $340 \mathrm{~nm}$. The activity of GPx was expressed in terms of nmol $\mathrm{NADPH}$ consumed $/ \mathrm{min} / \mathrm{mg}$ protein.

2.9.7. Determination of Glutathione Reductase (GR) Activity. The GR activity was determined by the method of KarMahapatra et al. [18]. The tubes for enzyme assay were incubated at $37^{\circ} \mathrm{C}$ and contained $2.0 \mathrm{~mL}$ of $9 \mathrm{mM}$ GSSG, $0.02 \mathrm{~mL}$ of $12 \mathrm{mM}$ NADPH, Na4, $2.68 \mathrm{~mL}$ of $1 / 15 \mathrm{M}$ phosphate buffer ( $\mathrm{pH}$ 6.6), and $0.1 \mathrm{~mL}$ of mitochondria. The activity of this enzyme was determined by monitoring the decrease in absorbance at $340 \mathrm{~nm}$. The activity of GR was expressed in terms of nmol NADPH consumed/min/mg protein.

2.9.8. Determination of Glutathione-S-Transferase (GST) Activity. The mitochondrial GST activity of liver and spleen was measured by the method of KarMahapatra et al. [18]. The tubes of enzyme assay were incubated at $25^{\circ} \mathrm{C}$ and contained $2.85 \mathrm{~mL}$ of $0.1 \mathrm{M}$ potassium phosphate ( $\mathrm{pH} 6.5$ ) containing $1 \mathrm{mM}$ of GSH, $0.05 \mathrm{~mL}$ of $60 \mathrm{mM}$ 1-chloro-2, 4-dinitrobengene and $0.1 \mathrm{~mL}$ of mitochondria from liver and spleen. The activity of this enzyme was determined by monitoring 
the increase in absorbance at $340 \mathrm{~nm}$. The activity of GST was expressed in terms of nmol NADPH consumed $/ \mathrm{min} / \mathrm{mg}$ protein.

2.9.9. Protein Estimation. Protein was determined using bovine serum albumin as a standard according to Lowry et al. [19].

2.9.10. Determination of Mitochondrial Membrane Potential $\left(\Delta \psi_{m}\right)$. Mitochondrial membrane potential was determined according to Reungpatthanaphong and Mankhetkorn [20] with some modification. Briefly, the hepatic and splenic mitochondria were incubated separately with $40 \mathrm{nM}$ rhodamine $B$ in $1 \mathrm{~cm}$ quartz cuvettes containing HEPES- $\mathrm{Na}^{+}$buffer, $\mathrm{pH} 7.3$ and vigorously stirred at $37^{\circ} \mathrm{C}$. The rhodamine $\mathrm{B}$ fluorescence at $582 \mathrm{~nm}$ (excited at $553 \mathrm{~nm}$ ) was monitored for 2 min using Hitachi F-7000 Fluorescence Spectrophotometer. Both excitation and emission slit width were set to 5.0.

2.10. Statistical Analysis. The data were expressed as mean \pm SEM, $n=6$. Comparisons between the means of control and infected group and also infected and treated group were made by Student's $t$-test (using a statistical package, Origin 6.1, Northampton, Mass, USA), $P<0.05$, level of significance.

\section{Results and Discussion}

3.1. Particle Characterization. The detail of the test sample characterization was already described in our previous report [14]. Therefore, we showed only the brief information about the sample characterization in the present report. FTIR spectra showed the most important peaks at $1565 \mathrm{~cm}^{-1}$, $2927 \mathrm{~cm}^{-1}$, and $1406 \mathrm{~cm}^{-1}$ which are assigned to $\mathrm{N}=\mathrm{O}$ stretching of amine presence in drug, $\mathrm{C}-\mathrm{H}$ stretching of methylene group, stretching vibrations of $\mathrm{C}=\mathrm{C}$, and $\mathrm{C}=\mathrm{N}$ which are on indicate presence of chloroquine on chitosan NPs, respectively. The particles size, measured by DLS, were in the range of $150-225 \mathrm{~nm}$, and the drug loaded nanoparticles size were in the range of $150-300 \mathrm{~nm}$. The highest fraction of chloroquine NP present in the solution was of less than $250 \mathrm{~nm}$, and the positive charge of $\mathrm{Nch}(+32.9 \mathrm{mV})$ is slightly higher than CS-TPP NPs. The cytotoxic activity of chitosanTPP nanoparticle on HeLa cell lines showed no significant difference in cell viability between the cells treated with chitosan-TPP NPs, and our previous study revealed that the paprticle has no activity on parasitemia alteration, and in the drug loaded particle, the actual drug content was approximately $27.36 \%$ and the encapsulation efficiency was over $54 \%$ of the nanoparticles [14].

3.2. Parasitemia. The pathology of malaria depends not only on the strain of Plasmodium but also on the species and strain of the vertebrate host [21,22]. Differences in the pathologies of Plasmodia strains appear to depend more on the strain of the parasite rather than on the type of mouse, indicating that the nature of the parasite and/or its antigens are more important than the mouse strain in determining the host defense response [22]. However, giemsa staining of tail vein blood smear was observed from control, infected, CQ treated, and Nch treated group to evaluate the parasitemia. In the

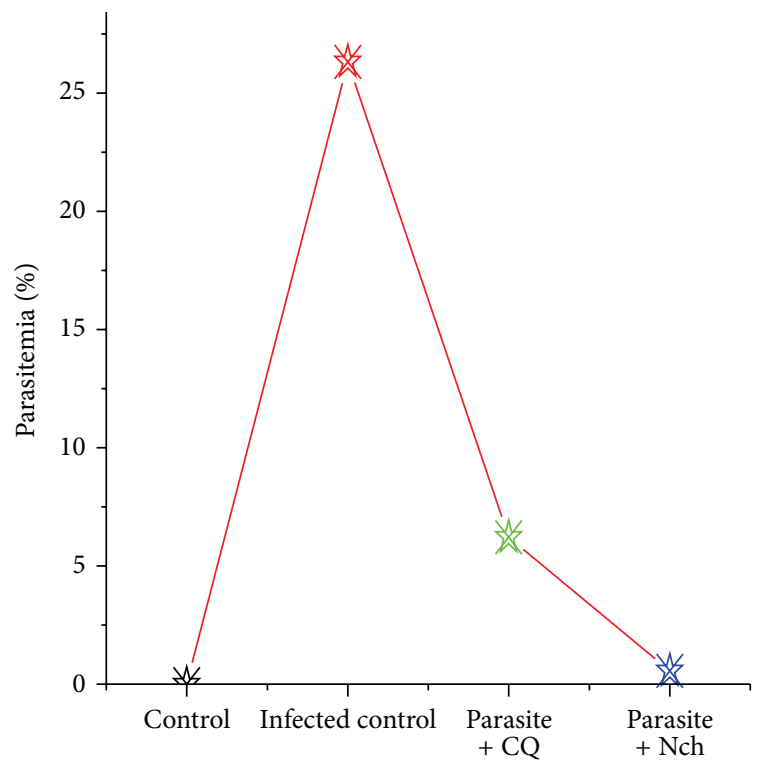

FIGURE 1: Graphical presentation of parasitemia level in different groups. Values are expressed as mean $\pm \operatorname{SEM}, n=6 .{ }^{*}$ Indicates the significant difference $(P<0.05)$ compared with control. ${ }^{*}$ Indicates the significant difference compared with infected group. Images are photographed at $\times 100$ magnification.

infected group, parasitemia was $26.32 \%$, whereas CQ treated group and Nch treated groups showed the parasitemia $6.21 \%$ and $0.56 \%$, respectively (Figure 1). It may be due to free heme which is a powerful free radical generator, which can cause serious molecular damage to both host and parasite; the heme group contains $\mathrm{Fe}^{2+}$ atoms that can catalyze Fenton and Haber-Weiss reactions, generating free radicals [23].

3.3. Superoxide Radical Generation. Alteration of the mitochondrial redox status by oxidative stress has been implicated in organ damage during malaria. Because mitochondria are the source and target of reactive oxygen species (ROS), we have investigated whether $P$. berghei is linked to mitochondrial pathology and mitochondrial ROS generation during malaria. It has been found that in the infected group the $\mathrm{O}_{2}{ }^{\circ}$ level increased significantly by $41.27 \%$ in liver mitochondria as well as in spleen it also increased by $52.37 \%$ but $\mathrm{Nch}$ treatment it decreased by $23.86 \%$ in liver mitochondria and $43.14 \%$ in spleen as compared to infected control (Figure 2).

3.4. Nitrate Generation. Nitrate (NO) is an indicator of free radical generation. NO levels were significantly $(P<0.05)$ increased in mitochondria of liver by $22.92 \%$, due to parasite infection as compared to control group, in which they were significantly $(P<0.05)$ decreased by $5.36 \%$ and $16.43 \%$ after treatment of CQ and Nch, respectively, as compared to infected control. In case of spleen mitochondria, the NO level in the infected group increased significantly $(P<0.05)$ by $38.48 \%$, and CQ treatment reduced the NO generation by $3.5 \%$, whereas Nch supplementation decreased by $10.5 \%$ as compared to infected control group. The results are shown in Figure 3. 


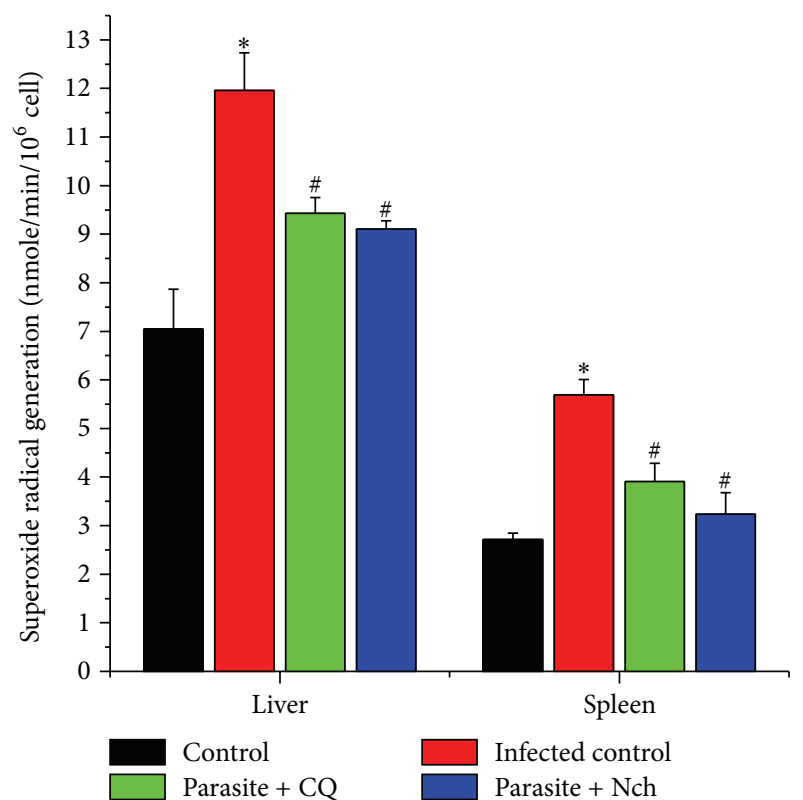

FIGURE 2: Graphical presentation of superoxide radical generation $\left(\mathrm{O}_{2}{ }^{\circ}\right)$ of hepatic and splenic mitochondria. Values are expressed as mean \pm SEM, $n=6 .{ }^{*}$ Indicates significant difference $(P<0.05)$ compared to control group. ${ }^{*}$ Indicates significant difference $(P<$ 0.05) compared to infected group.

3.5. Superoxide Dismutase (SOD) Activity. SOD, a chain breaking antioxidant, plays an important role in protection against the deleterious effects of lipid peroxidation [24]. Free radicals such as partially reduced and highly reactive oxygen species $\left(\mathrm{O}_{2}\right), \mathrm{H}_{2} \mathrm{O}_{2}$ or hydrogen peroxide, hydroxyl radical $\left(\mathrm{OH}^{-}\right)$, and singlet oxygen have been implicated in the etiology of various diseases. SOD is the major enzyme that protects cells from damage by catalyzing the dismutation of the superoxide into oxygen and hydrogen peroxide. From this study, the SOD generation in mitochondria, SOD level is decreased in liver mitochondria by $76.4 \%$ as compared to control due to infection of $P$. berghei, but in Nch treated group it has been increased significantly, similarly, the SOD level in splenic mitochondria also decreased in infected group by $47.59 \%$ and significantly $(P<0.05)$ increased in Nch treated group by $41.11 \%$ than CQ $(39.16 \%)$ treated group as compared to infected control. The results are shown in Figure 4.

\subsection{Reduced Glutathione (GSH) and Oxidized Glutathione} (GSSG). Glutathione is an important antioxidant in cellular system. So, to understand glutathione level, we have measured both reduced and oxidized form of glutathione. The reduced glutathione level was decreased significantly $(P<$ 0.05 ) by $53.24 \%$ in liver of infected group, as compared to control, whereas the oxidized glutathione level was increased significantly $(P<0.05)$ by $109.77 \%$, as compared to control. Treatment of nanodrug significantly $(P<0.05)$ increased GSH level by $52.44 \%$ and decreased GSSG level significantly $(P<0.05)$ by $48.93 \%$ in liver. Similarly, the Nch supplementation increased the GSH level in splenic mitochondria

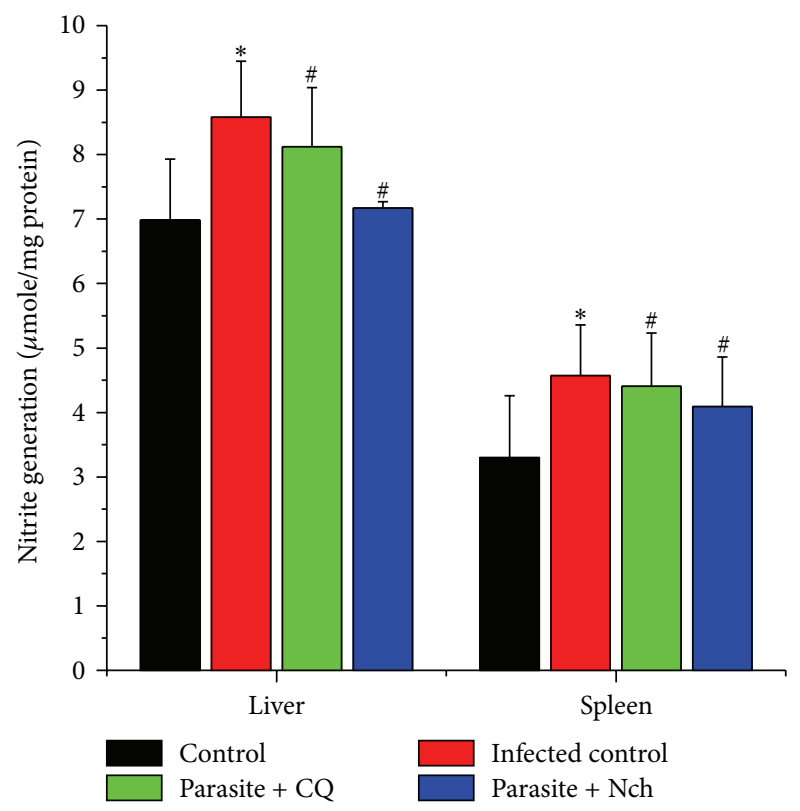

FIGURE 3: Graphical presentation of nitrite generation (NO) of hepatic and splenic mitochondria. Values are expressed as mean \pm SEM, $n=6 .{ }^{*}$ Indicates significant difference $(P<0.05)$ compared to control group. ${ }^{\#}$ indicates significant difference $(P<0.05)$ compared to infected group.

as compared to infected control has been shown in Figures 5 and 6.

3.7. Glutathione Reductase (GR) and Glutathione-S-Transferase (GST) Activity. Many enzymes are important for synthesis, turnover, and maintenance of intracellular GSH concentrations [23, 24]. For the purpose of this paper, we term GSH reductase (GR), and glutathione-S-transferases (GSTs) was measured to understand the antioxidant enzyme activity of different experimental mitochondria. GR activity is significantly $(P<0.05)$ diminished in liver, in case of $P$. berghei infected group in Figure 7. It was recovered after nanoconjugated chloroquine treatment which increased antioxidant enzyme activity in mitochondria. Glutathione transferase (GST) is also measured to understand the antioxidant enzyme activity. Nanoconjugated chloroquine protects the mitochondria to elevate antioxidant enzyme activity to keep the organelles safe from oxidative damage due to malaria. The results are shown in Figure 8.

3.8. Mitochondrial Membrane Potential $\left(\Delta \psi_{m}\right)$. The result in Figure 9 shows that the P. berghei infection decreased $\psi m$, as evidenced from $40.74 \%$ and $33.66 \%$ reduction in hepatic and splenic mitochondria then control in fluorescence at $582 \mathrm{~nm}$ due to rhodamine b uptake $(P<0.05)$, whereas in $\mathrm{Nch}$ treated group showed the membrane potential increment significantly $(P<0.05)$ then infected group.

Recently, biodegradable and biocompatible polymeric chitosan nanoparticles have attracted a considerable attention as potential carriers for the controlled and site-specific delivery of drugs [25]. Due to its unique polymeric cationic cha- 


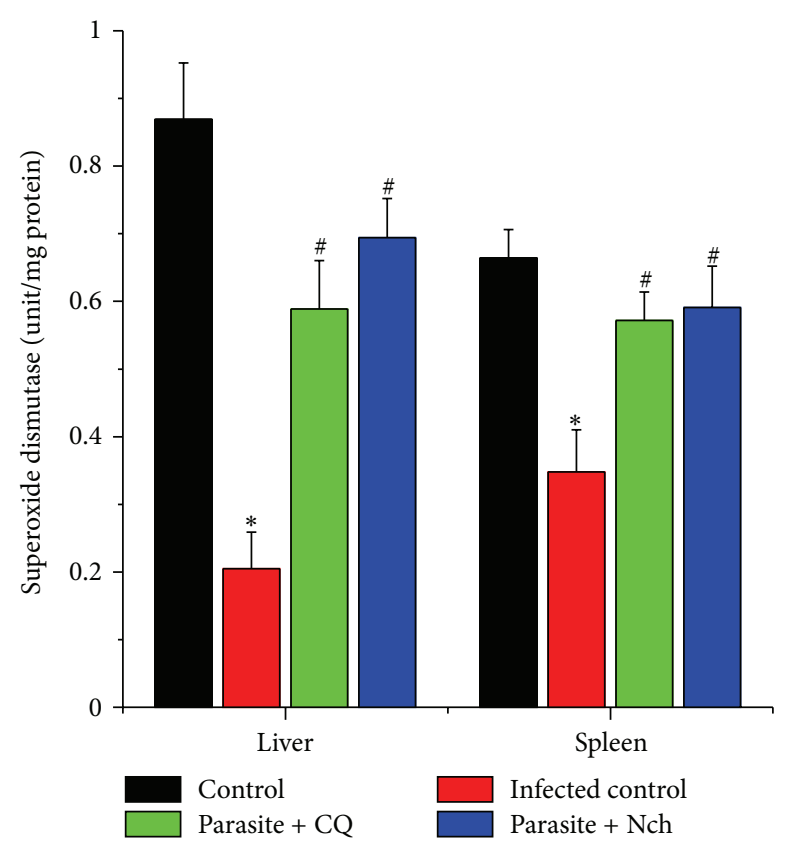

FIGURE 4: Graphical presentation of superoxide dismutase (SOD) of hepatic and splenic mitochondria. Values are expressed as mean \pm SEM, $n=6 .{ }^{*}$ Indicates significant difference $(P<0.05)$ compared to control group. ${ }^{\#}$ Indicates significant difference $(P<0.05)$ compared to infected group.

racter, CS has been extensively examined for the development of drug delivery systems in the pharmaceutical industry [26]. Due to the availability of free amino groups, it carries a positive charge and reacts with many negatively charged surfaces of the cell membrane, mucus lining (due to negatively charged sialic acid residues), and also with other anionic polymers. The nanodrug delivery systems seem to be a promising and viable approach for improving malaria treatment [27]. Plasmodium infection leads to increased oxidative stress in the vertebrate hosts. The high proliferation rate of parasites results in the production of large quantities of toxic redoxactive by-products [28]. The role of oxidative stress during malarial infection needs clear illumination, as some have demonstrated a protective role while others suggested a relation with malarial pathophysiology and complication [29].

ROS are generated as by-products of aerobic respiration and various other catabolic and anabolic processes. Mitochondria are the major producer of ROS in cells, and the bulk of mitochondrial ROS is generated at the electron transport chain. Electrons leak from the electron transport chain directly to oxygen, producing short-lived free radicals such as superoxide anion $\left(\mathrm{O}_{2}{ }^{-}\right) \cdot \mathrm{O}_{2}{ }^{-}$can be converted to nonradical derivatives such as hydrogen peroxide $\left(\mathrm{H}_{2} \mathrm{O}_{2}\right)$ either spontaneously or catalyzed by superoxide dismutase (SOD). $\mathrm{H}_{2} \mathrm{O}_{2}$ is relatively stable and membrane permeable. It can be diffused within the cell and be removed by cytosolic antioxidant systems such as catalase, glutathione peroxidase. Because mitochondria are the major producer of ROS in mammalian cells, the close proximity to ROS places mitochondrial DNA (mtDNA) prone to oxidative damage. Consistently, many

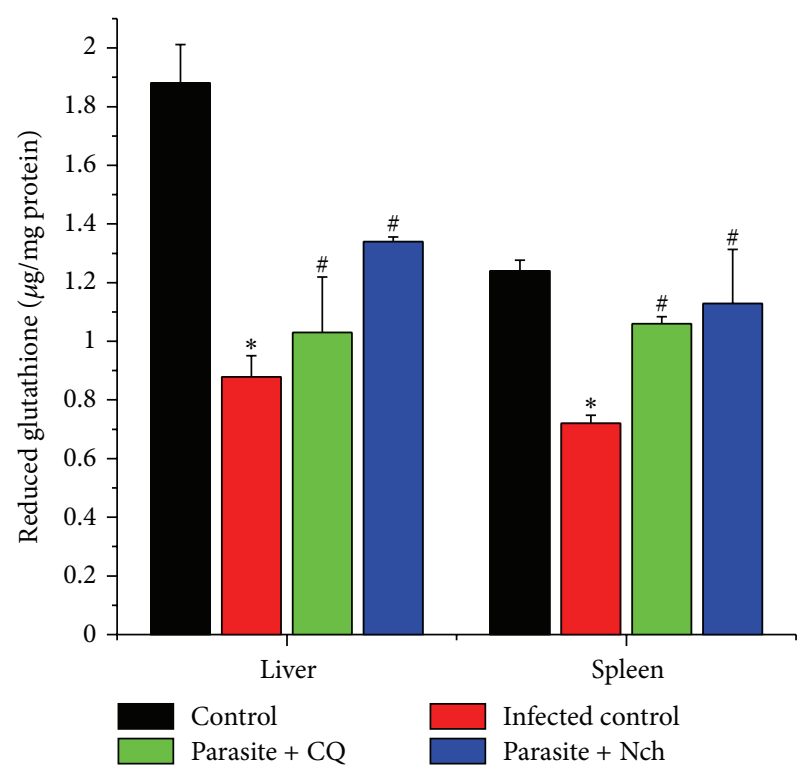

FIGURE 5: Graphical presentation of reduced glutathione (GSH) level of hepatic and splenic mitochondria. Values are expressed as mean \pm SEM, $n=6 .{ }^{*}$ Indicates significant difference $(P<0.05)$ compared to control group. "Indicates significant difference $(P<$ 0.05) compared to infected group.

studies have shown that 8-oxo-dG, one of the common oxidative lesions, is detected at higher level in mtDNA than nuclear DNA, suggesting that mtDNA is more susceptible to oxidative damage [24].

In the study, it is reported that during the erythrocytic phase of malaria, red blood cell lysis and the release of hemozoin occur, which consists primarily of ferriprotoporphyrin IX dimmers and monomers (FP) and methemoglobin in plasmodial proteins. Free heme is a powerful free radical generator, which can cause serious molecular damage to both host and parasite; the heme group contains $\mathrm{Fe}^{2+}$ atoms that can catalyze Fenton and Haber-Weiss reactions, generating free radicals [30]. This study demonstrates that infection of mice with Plasmodium berghei malaria produces demonstrable disturbance in the behavior of hepatic and splenic mitochondria and causes cell damage due to $\mathrm{O}_{2}{ }^{--}$and NO production as well as antioxidant enzyme reduction where Nch treatment group showed the increment of $\psi \mathrm{m}$. Malarial infection induces mitochondrial pathology by reducing transmembrane potential. Mitochondrial GSH depletion and formation of $\mathrm{O}_{2}{ }^{--}$indicate that mitochondrial pathology is associated with mitochondrial oxidative stress intramitochondrial $\mathrm{O}_{2}{ }^{\cdot-}$ generation during malaria. $\mathrm{O}_{2}{ }^{--}$inactivates mitochondrial aconitase to release iron from iron-sulfur clusters, which forms the hydroxyl radical. $\mathrm{OH}$ interacting with $\mathrm{H}_{2} \mathrm{O}_{2}$ produced concurrently [8].

Oxidative stress is known to induce apoptosis in many cellular systems, including liver [30-32] and spleen [33]. It has been reported that $P$. yoelii infection is responsible to induce opening of MPTP, as a result of that mitochondrial membrane permeability increases, leading to a decrease in $\psi \mathrm{m}$. Mitochondrial anomalies and upregulation of apoptosis inducing 


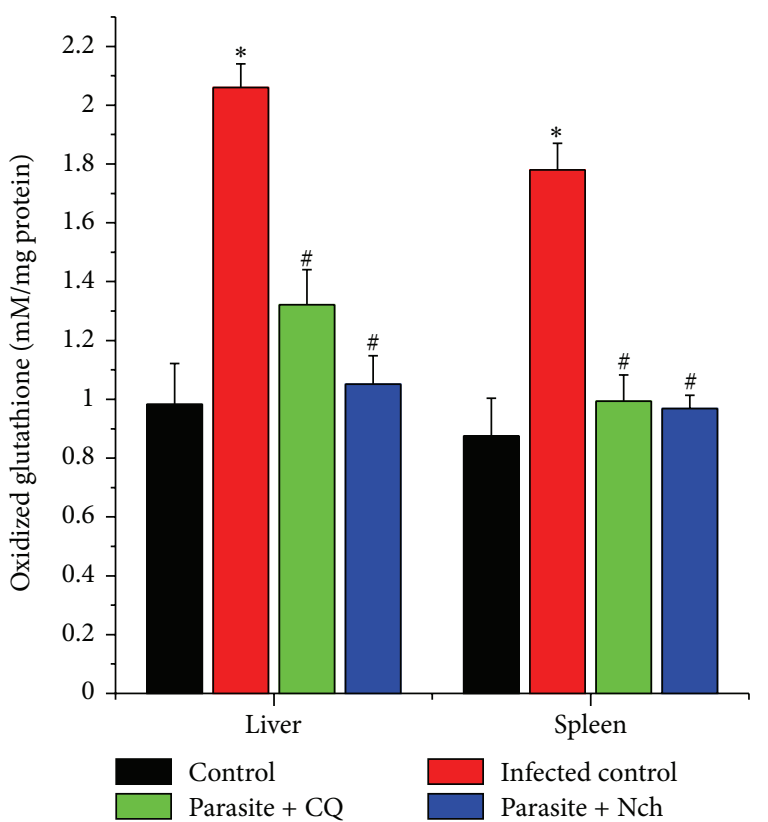

FIGURE 6: Graphical presentation of oxidized glutathione (GSSG) level of hepatic and splenic mitochondria. Values are expressed as mean \pm SEM, $n=6$. ${ }^{*}$ Indicates significant difference $(P<0.05)$ compared to control group. ${ }^{*}$ Indicates significant difference $(P<$ 0.05) compared to infected group.

proteins such as Bax, p53 have also been reported in mice brain during experimental murine cerebral malaria. Opening of MPTP causes release of cytochrome $c$ from mitochondria to cytosol [34, 35]. Generation of ROS and associated oxidative stress has been found to activate mitochondrial pathway of apoptosis [36]. We have found that P. berghei infection significantly induces $\mathrm{OH}$ generation in the liver and spleen of infected mice. Scavenging of ${ }^{-} \mathrm{OH}$ also significantly inhibits the mitochondrial pathway, as evidenced from the near normal restoration of $\psi m$ [37]. The data suggest that administration of Nch attenuates $P$. berghei infection associated oxidative stress (assayed by lipid peroxidation) as well as apoptosis (as revealed from reduction of membrane potential), indicating a causative role of ${ }^{-} \mathrm{OH}$ in the initiation of apoptosis. As mitochondria play a critical role in regulation of apoptosis, which is implicated in the pathogenic infection, the activation of the permeability transition pore in mitochondria is believed to play a critical role in cell necrosis and apoptosis [38]. Such selective oxidative modification may cause the cells to be more vulnerable to cell damage inducers.

\section{Conclusion}

From this study it may conclude that, chitosan-tripolyphosphate conjugated chloroquine nanoparticles potentially able to protect the mitochondrial damage of liver as well as defend the spleen from oxidative damage due to $P$. berghei infection by decreasing free radical generation and also by increasing the antioxidant enzymes level. The nanoconjugated drug may become the focus in future as a new therapeutic antimalarial agent as well as a potent free radical

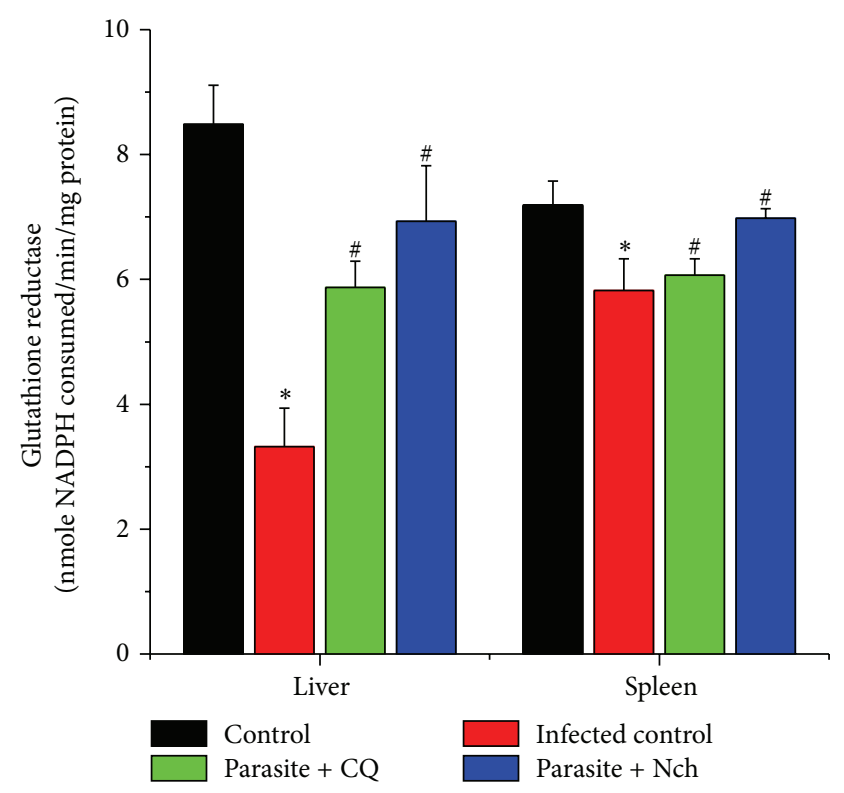

FIGURE 7: Graphical presentation of glutathione reductase (GR) activity of hepatic and splenic mitochondria. Values are expressed as mean \pm SEM, $n=6$. ${ }^{*}$ Indicates significant difference $(P<0.05)$ compared to control group. "Indicates significant difference $(P<$ 0.05) compared to infected group.

scavenger against such infection. However, further critical studies are necessary to clarify the mechanism of activity and also its role against chloroquine resistant parasite, including immune mechanisms in the course of medication.
Abbreviations
bw: Body weight
DLS: Dynamic light scattering
FTIR: $\quad$ Fourier transform infrared spectroscopy
DTNB: $\quad 5^{\prime}, 5^{\prime}$-Dithio (bis)-2-nitrobenzoic acid
EDTA: Ethylene diamine tetra acetate
GR: $\quad$ Glutathione reductase
GSH: $\quad$ Reduced glutathione
GSSG: Oxidized glutathione
$\mathrm{H}_{2} \mathrm{O}_{2}$ : Hydrogen peroxide
MDA: Malondialdehyde
MPO: Myeloperoxidase
NADPH: Nicotinamide adenine dinucleotide phosphate
Nch: Nanochloroquine
OPD: O-Dianisidine
PBS: Phosphate buffer saline
ROS: Reactive oxygen species
TBA: Thiobarbituric acid
TCA: Trichloroacetic acid.

\section{Conflict of Interest}

The authors declare that there is no conflicts of interests. 


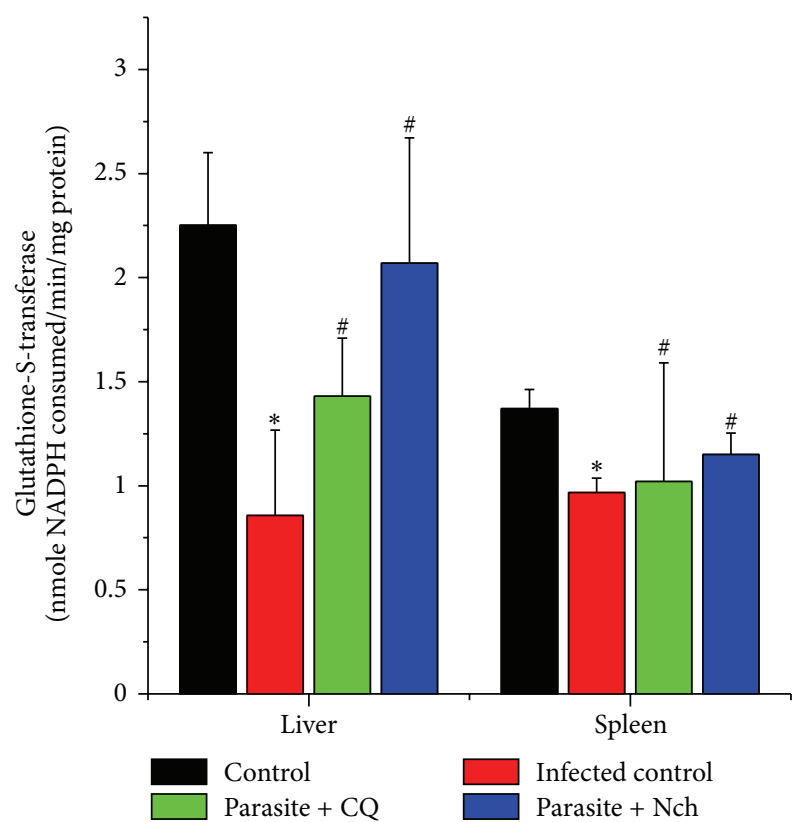

FIGURE 8: Graphical presentation of glutathione-s-transferase (GST) activity of hepatic and splenic mitochondria. Values are expressed as mean \pm SEM, $n=6 .{ }^{*}$ Indicates significant difference $(P<0.05)$ compared to control group. ${ }^{*}$ Indicates significant difference $(P<$ $0.05)$ compared to infected group.

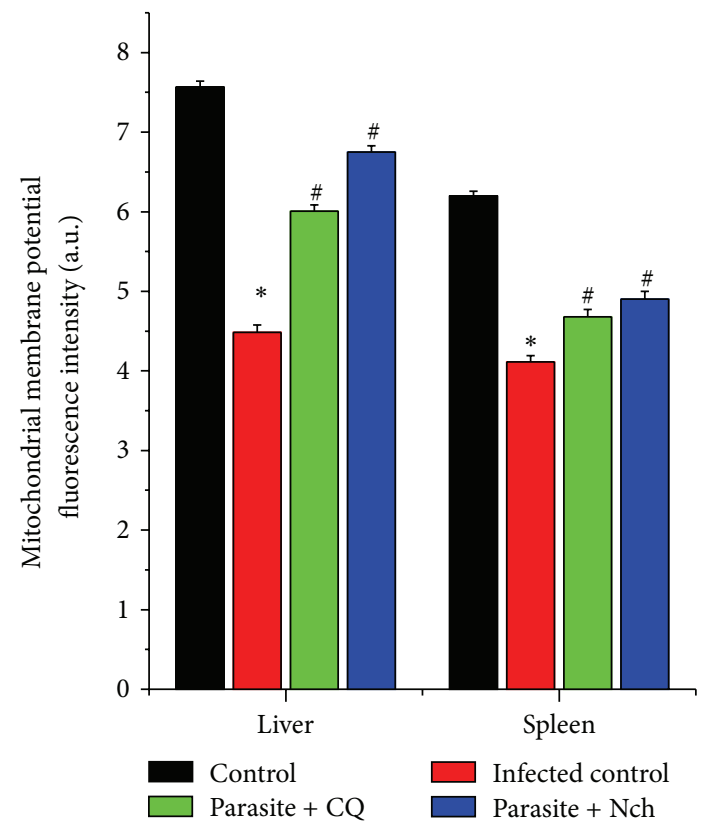

Figure 9: Graphical presentation of mitochondrial membrane potential alteration. Values are expressed as mean \pm SEM, $n=6$. ${ }^{*}$ Indicates significant difference $(P<0.05)$ compared to control group. ${ }^{\#}$ Indicates significant difference $(P<0.05)$ compared to infected group.

\section{Acknowledgments}

The authors thank Professor Pralhad C. Ghosh (Delhi University, South Campus, Delhi, India) for supplying the parasite strain and authors express gratitude to the Indian Institute of Technology, Kharagpur 721302, and Vidyasagar University, Midnapore 721102, for providing the facilities to execute these studies.

\section{References}

[1] P. L. Olliaro and P. I. Trigg, "Status of antimalarial drugs under development," Bulletin of the World Health Organization, vol. 73, no. 5, pp. 565-571, 1995.

[2] W. Peters, "The problem of drug resistance in malaria," Parasitology, vol. 90, pp. 705-715, 1985.

[3] N. J. White, "Antimalarial drug resistance: the pace quickens," Journal of Antimicrobial Chemotherapy, vol. 30, no. 5, pp. 571585, 1992.

[4] L. R. Brunet, "Nitric oxide in parasitic infections," International Immunopharmacology, vol. 1, no. 8, pp. 1457-1467, 2001.

[5] M. M. Stevenson and E. M. Riley, "Innate immunity to malaria," Nature Reviews Immunology, vol. 4, no. 3, pp. 169-180, 2004.

[6] A. Holmgren, "Antioxidant function of thioredoxin and glutaredoxin systems," Antioxidants and Redox Signaling, vol. 2, no. 4, pp. 811-820, 2000.

[7] P. Srivastava, S. K. Puri, G. P. Dutta, and V. C. Pandey, "Hepatic superoxide-scavenging system during Plasmodium berghei infection and chloroquine treatment," Medical Science Research, vol. 19, no. 10, pp. 307-308, 1991.

[8] C. Guinovart, M. M. Navia, M. Tanner, and P. L. Alonso, "Malaria: burden of disease," Current Molecular Medicine, vol. 6, no. 2, pp. 137-140, 2006.

[9] S. Dey, M. Guha, A. Alam et al., "Malarial infection develops mitochondrial pathology and mitochondrial oxidative stress to promote hepatocyte apoptosis," Free Radical Biology and Medicine, vol. 46, no. 2, pp. 271-281, 2009.

[10] I. Landau and P. Gautret, "Animal models: rodents," in Malaria: Parasite Biology, Pathogenesis, and Protection, I. W. Sherman, Ed., pp. 401-417, ASM Press, Washington, DC, USA, 1998.

[11] W. Peters, J. H. Portus, and B. L. Robinson, "The chemotherapy of rodent malaria, XXII. The value of drug resistant strains of $P$. berghei in screening for blood schizontocidal activity," Annals of Tropical Medicine and Parasitology, vol. 69, no. 2, pp. 155-171, 1975.

[12] E. C. Ibezim, C. T. Andrade, C. M. B. Barretto, D. C. Odimegwu, and F. F. D. Lima, "Ionically cross-linked chitosan/tripolyphosphate microparticles for the controlled delivery of pyrimethamine," Ibinosina Journal of Medicine and Biological Sciences, vol. 3, no. 3, pp. 77-87, 2011.

[13] J. W. Loh, M. Saunders, and L. Y. Lim, "Cytotoxicity of monodispersed chitosan nanoparticles against the Caco-2 cells," Toxicology and Applied Pharmacology, vol. 262, no. 3, pp. 273-282, 2012.

[14] S. Tripathy, S. Das, S. P. Chakraborty, S. K. Sahu, P. Pramanik, and S. Roy, "Synthesis, characterization of chitosan-tripolyphosphate conjugated chloroquine nanoparticle and its in vivo anti-malarial efficacy against rodent parasite: a dose and duration dependent approach," International Journal of Pharmaceutics, vol. 434, pp. 292-305, 2012.

[15] S. Tripathy, S. P. Chakraborty, and S. Roy, "Superoxide radical generation mediated Plasmodium berghei infection in Swiss mice," Al Ameen Journal of Medical Sciences, vol. 5, pp. 69-81, 2012. 
[16] J. R. Aprille and J. Austin, "Regulation of the mitochondrial adenine nucleotide pool size," Archives of Biochemistry and Biophysics, vol. 212, no. 2, pp. 689-699, 1981.

[17] A. Boveris, "Determination of the production of superoxide radicals and hydrogen peroxide in mitochondria," Methods in Enzymology, vol. 105, pp. 429-435, 1984.

[18] S. KarMahapatra, S. P. Chakraborty, S. Das, and S. Roy, "Methanol extract of Ocimum gratissimum protects murine peritoneal macrophages from nicotine toxicity by decreasing free radical generation, lipid and protein damage and enhances antioxidant protection," Oxidative Medicine and Cellular Longevity, vol. 2, no. 4, pp. 222-230, 2009.

[19] O. H. Lowry, N. J. Rosenbrough, A. L. Farr, and R. J. Randall, "Protein measurement with the Folin phenol reagent," The Journal of Biological Chemistry, vol. 193, no. 1, pp. 265-275, 1951.

[20] P. Reungpatthanaphong and S. Mankhetkorn, "Modulation of multidrug resistance by artemisinin, artesunate and dihydroartemisinin in K562/adr and GLC4/adr resistant cell lines," Biological and Pharmaceutical Bulletin, vol. 25, no. 12, pp. 15551561, 2002.

[21] B. R. Moore, J. D. Jago, and K. T. Batty, "Plasmodium berghei: parasite clearance after treatment with dihydroartemisinin in an asplenic murine malaria model," Experimental Parasitology, vol. 118, no. 4, pp. 458-467, 2008.

[22] R. Carter and D. Walliker, "New observations on the malaria parasites of rodents of the Central African Republic. Plasmodium vinckei petteri subsp. nov. and Plasmodium chabaudi Landau, 1965," Annals of Tropical Medicine and Parasitology, vol. 69, no. 2, pp. 187-196, 1975.

[23] B. S. Das and N. K. Nanda, "Evidence for erythrocyte lipid pepoxidation in acute falciparum malaria," Transactions of the Royal Society of Tropical Medicine and Hygiene, vol. 93, no. 1, pp. 5862, 1999.

[24] J. H. Santos, B. S. Mandavilli, and B. Van Houten, "Measuring oxidative mtDNA damage and repair using quantitative PCR," Methods in Molecular Biology, vol. 197, pp. 159-176, 2002.

[25] N. Evelyne, P. Nathaline, F. Candau et al., "Biopolymer and polymer nanoparticles and their biomedical applications," Handbook of Nanostructure and Nanotechnology, vol. 5, pp. 577-635, 2000.

[26] K. A. Janes, P. Calvo, and M. J. Alonso, "Polysaccharide colloidal particles as delivery systems for macromolecules," Advanced Drug Delivery Reviews, vol. 47, no. 1, pp. 83-97, 2001.

[27] H. Swai, B. Semete, L. Kalombo, and P. Chelule, "Potential of treating tuberculosis with a polymeric nano-drug delivery system," Journal of Controlled Release, vol. 132, p. e48, 2008.

[28] I. Landau and A. G. Chabaud, "Natural infection by 2 plasmodia of the rodent Thamnomys rutilans in the Central African Republic," Comptes Rendus Hebdomadaires des Seances de l"Academie des Sciences D, vol. 261, no. 1, pp. 230-232, 1965.

[29] M. Yoeli, B. Hargreaves, R. Carter, and D. Walliker, "Sudden increase in virulence in a strain of Plasmodium yoelii," Annals of Tropical Medicine and Parasitology, vol. 69, no. 2, pp. 173-178, 1975.

[30] B. S. Das and N. K. Nanda, "Evidence for erythrocyte lipid pepoxidation in acute falciparum malaria," Transactions of the Royal Society of Tropical Medicine and Hygiene, vol. 93, no. 1, pp. 5862, 1999.

[31] A. Pabon, J. Carmona, L. C. Burgos, and S. Blair, "Oxidative stress in patients with non-complicated malaria," Clinical Biochemistry, vol. 36, pp. 71-78, 2003.
[32] M. J. Czaja, "Induction and regulation of hepatocyte apoptosis by oxidative stress," Antioxidants and Redox Signaling, vol. 4, no. 5, pp. 759-767, 2002.

[33] T. A. Sarafian and D. E. Bredesen, "Is apoptosis mediated by reactive oxygen species?” Free Radical Research, vol. 21, no. 1, pp. 1-8, 1994.

[34] K. Biswas, U. Bandyopadhyay, I. Chattopadhyay, A. Varadaraj, E. Ali, and R. K. Banerjee, "A novel antioxidant and antiapoptotic role of omeprazole to block gastric ulcer through scavenging of hydroxyl radical," Journal of Biological Chemistry, vol. 278, no. 13, pp. 10993-11001, 2003.

[35] D. R. Green and J. C. Reed, "Mitochondria and apoptosis," Science, vol. 281, no. 5381, pp. 1309-1312, 1998.

[36] H. U. Simon, A. Haj-Yehia, and F. Levi-Schaffer, "Role of reactive oxygen species (ROS) in apoptosis induction," Apoptosis, vol. 5, no. 5, pp. 415-418, 2000.

[37] M. Guha, S. Kumar, V. Choubey, P. Maity, and U. Bandyopadhyay, "Apoptosis in liver during malaria: role of oxidative stress and implication of mitochondrial pathway," The FASEB Journal, vol. 20, no. 8, pp. E439-E449, 2006.

[38] L. Sanchez-Torres, A. Rodriguez-Ropon, M. Aguilar-Medina, and L. Favila-Castillo, "Mouse splenic $\mathrm{CD}^{+}$and $\mathrm{CD}^{+} \mathrm{T}$ cells undergo extensive apoptosis during a Plasmodium chabaudi chabaudi AS infection," Parasite Immunology, vol. 23, no. 12, pp. 617-626, 2001. 

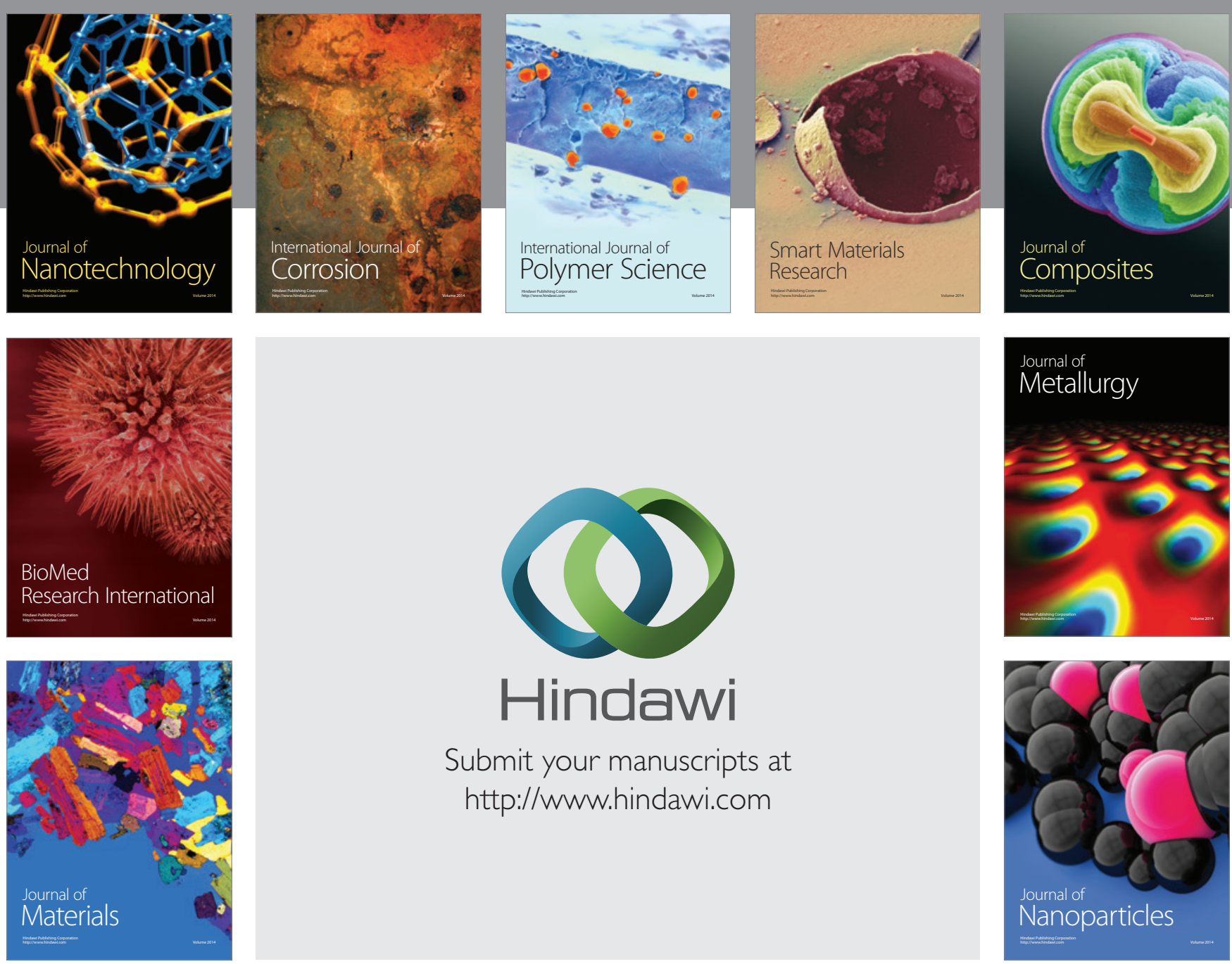

Submit your manuscripts at http://www.hindawi.com
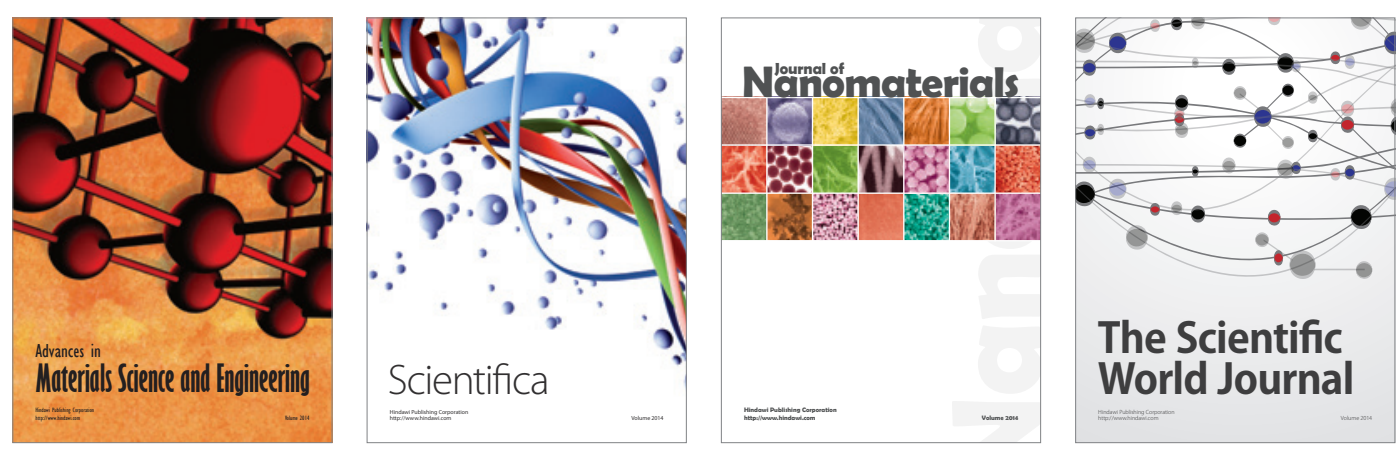

\section{The Scientific World Journal}
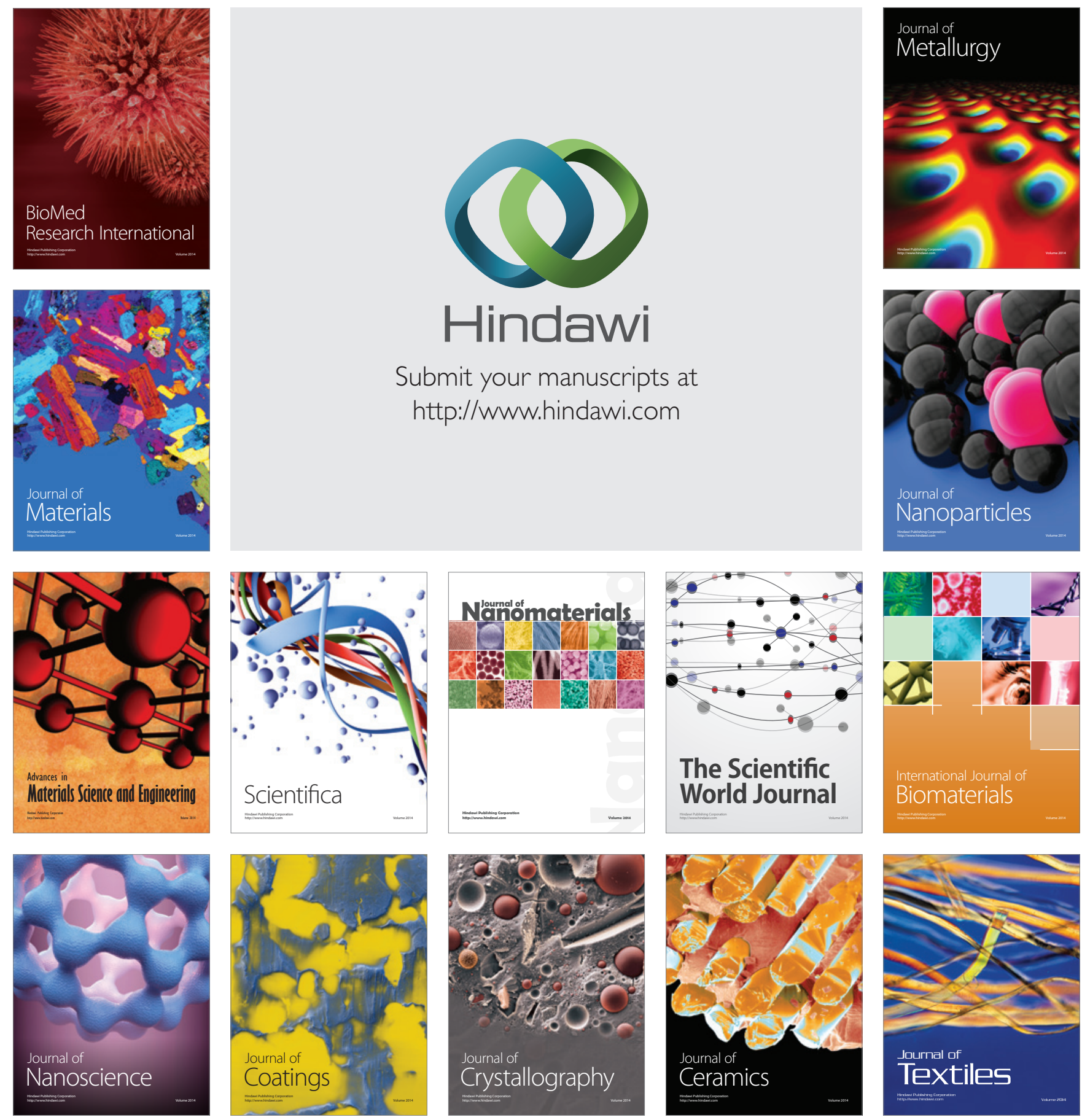